\title{
Holistic care program for elderly patients to integrate spiritual needs, social activity, and self-care into disease management in primary care (HoPES3): study protocol for a cluster-randomized trial
}

Cornelia Straßner ${ }^{1 *}$ D Eckhard Frick², Gabriele Stotz-Ingenlath², Nicola Buhlinger-Göpfarth ${ }^{1}$, Joachim Szecsenyi ${ }^{1}$, Johannes Krisam ${ }^{3}$, Friederike Schalhorn ${ }^{4}$, Jan Valentini ${ }^{4}$, Regina Stolz ${ }^{4}$ and Stefanie Joos ${ }^{4}$

\begin{abstract}
Background: Strategies to improve the care of elderly, multimorbid patients frequently focus on implementing evidence-based knowledge by structured assessments and standardization of care. In Germany, disease management programs (DMPs), for example, are run by general practitioners (GPs) for this purpose. While the importance of such measures is undeniable, there is a risk of ignoring other dimensions of care which are essential, especially for elderly patients: their spiritual needs and personal resources, loneliness and social integration, and self-care (i.e., the ability of patients to do something on their own except taking medications to increase their well-being). The aim of this study is to explore whether combining DMPs with interventions to address these dimensions is feasible and has any impact on relevant outcomes in elderly patients with polypharmacy.

(Continued on next page)
\end{abstract}

\footnotetext{
* Correspondence: cornelia.strassner@med.uni-heidelberg.de

${ }^{1}$ Department of General Practice and Health Services Research, University

Hospital Heidelberg, Im Neuenheimer Feld 130.3, 69120 Heidelberg,

Germany

Full list of author information is available at the end of the article
}

(c) The Author(s). 2019 Open Access This article is distributed under the terms of the Creative Commons Attribution 4.0 International License (http://creativecommons.org/licenses/by/4.0/), which permits unrestricted use, distribution, and reproduction in any medium, provided you give appropriate credit to the original author(s) and the source, provide a link to the Creative Commons license, and indicate if changes were made. The Creative Commons Public Domain Dedication waiver (http://creativecommons.org/publicdomain/zero/1.0/) applies to the data made available in this article, unless otherwise stated. 


\begin{abstract}
(Continued from previous page)
Methods: An explorative, cluster-randomized controlled trial with general practices as the unit of randomization will be conducted and accompanied by a process evaluation. Patients aged 70 years or older with at least three chronic conditions receiving at least three medications participating in at least one DMP will be included. The control group will receive DMP as usual. In the intervention group, GPs will conduct a spiritual needs assessment during the routinely planned DMP appointments and explore whether the patient has a need for more social contact or self-care. To enable GPs to react to such needs, several aids will be provided by the study: a) training of GPs in spiritual needs assessment and training of medical assistants in patient counseling regarding self-care and social activity; b) access to a summary of regional social offers for seniors; and c) information leaflets on nonpharmacological interventions (e.g., home remedies) to be applied by patients themselves to reduce frequent symptoms in old age. The primary outcome is health-related self-efficacy (using the Self-Efficacy for Managing Chronic Disease 6-Item Scale (SES-6G)). Secondary outcomes are general self-efficacy (using the General Self-Efficacy Scale (GSES)), physical and mental health (using the Short-Form Health Survey (SF-12)), patient activation (using the Patient Activation Measure (PAM)), medication adherence (using the Medication Adherence Report Scale (MARS)), beliefs in medicine (using the Beliefs About Medicines Questionnaire (BMQ)), satisfaction with GP care (using selected items of the European Project on Patient Evaluation of General Practice (EUROPEP)), social contacts (using the 6-item Lubben Social Network Scale (LSNS-6)), and loneliness (using the 11-item De-Jong-Gierveld Loneliness Scale (DJGS-11)). Interviews will be conducted to assess the mechanisms, feasibility, and acceptability of the interventions.
\end{abstract}

Discussion: If the interventions prove to be effective and feasible, large-scale implementation should be sought and evaluated by a confirmatory design.

Trial registration: German Clinical Trials Register (DRKS), DRKS00015696. Registered on 22 January 2019.

Keywords: Spiritual care, Primary care, Loneliness, Medication, Old age, Home remedies, Naturopathic medicine, Alternative medicine, Self-efficacy, Self-care

\section{Background}

In most industrial countries the proportion of older people in the general population is constantly increasing due to lower birth rates and improved medical treatment options. In Europe, the mean life expectancy is currently 83.1 years for women and 77.5 years for men. About $20 \%$ of the European population is older than 65 years and this trend is rising since the fertility rate is 1.6 children per European woman, lower than necessary to keep the population stable [1]. Elderly people frequently suffer from multiple chronic diseases and are consequently treated with polypharmacy. Across Europe, 31\% of older adults take five medications or more per day [2]. Multimorbid patients with polypharmacy have a higher risk for potentially avoidable hospitalizations and adverse drug reactions causing a substantial proportion of healthcare costs [3, 4]. Furthermore, they experience significantly lower quality of life than those with single diseases or taking fewer drugs [5].

Because of this, elderly patients with multimorbidity and polypharmacy have been receiving increasing attention from healthcare research and health policies. Interventions to improve their care frequently focus on medication safety, the prevention of delirium and falls, or the maintenance of mobility and pain control. Common strategies to achieve these goals are the provision of knowledge (e.g., by involving pharmacists into care), comprehensive geriatric assessments, or disease and case management $[6,7]$. In Germany, disease management programs (DMPs) for individuals with diabetes, coronary heart disease and asthma/chronic obstructive pulmonary disease (COPD) are run by general practitioners (GPs). The core intervention of DMPs are regular appointments every 3-6 months during which a treatment protocol has to be completed.

Although the value of implementing standards and evidence-based knowledge into healthcare is undeniable, there is a risk of focusing healthcare for elderly patients too much on standardized measures overlooking other, more individual dimensions of care which also influence perceived quality of life. In a large German survey among elderly patients, self-efficacy was the most important determinant for well-being [8]. Self-efficacy can be defined as a person's estimate of his or her own ability to succeed in reaching a specific goal, e.g., reducing symptoms or maintaining good health. Consequently, strengthening patients' self-efficacy has the potential to substantially improve their quality of life. Furthermore, qualitative and quantitative research suggests that stronger self-efficacy is associated with better medication adherence [9-11].

As Fig. 1 shows, the intervention evaluated in this study consists of three major components addressing three interlinked domains related to self-efficacy (spiritual needs, social activity, and self-care) on which we will elaborate below. 


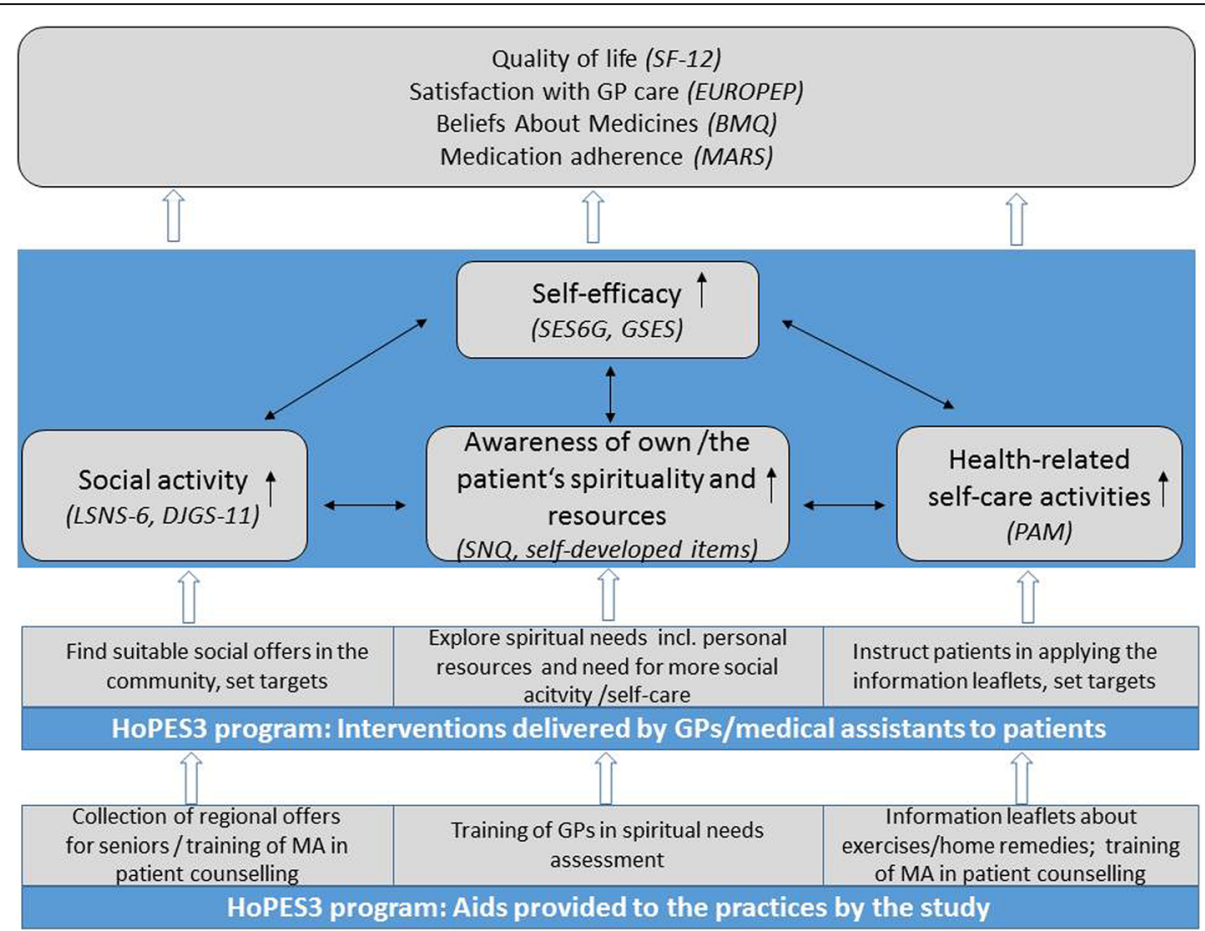

Fig. 1 Logic model of the HoPES3 program. BMQ Beliefs About Medicines Questionnaire, DJGS-11 11 -Item De-Jong-Gierveld Loneliness Scale, EUROPEP European Project on Patient Evaluation of General Practice, GP general practitioner, GSES General Self-Efficacy Scale, LSNS-6 6-Item Lubben Social Network Scale, MA medical Assistant, MARS Medication Adherence Report Scale, PAM Patient Activation Measure, SES6G SelfEfficacy for Managing Chronic Disease 6-Item Scale, SF-12 Short-Form Health Survey, SNQ Spiritual Needs Questionnaire

\section{Spiritual needs}

To be able to strengthen patients' self-efficacy it is necessary to be aware of their personal resources and their spirituality. The conceptual definitions of spirituality vary considerably [12]. In the context of healthcare, it is important to understand that spirituality is considered a dimension of the human being meaning that all humans have spiritual needs which differ in form and content. For this study, we define spiritual needs as everything which gives meaning to a person's life and thus serves as a personal resource.

A representative survey among Germans revealed that the individual factors determining meaning of life change with age. While younger persons mentioned "friends", "partnership", or "work" as decisive factors, religious and nature experiences were most important to patients aged 70 or older [13]. A growing body of literature suggests connections between satisfaction of spiritual needs and mental and physical health [14]. It has been substantiated for a broad spectrum of conditions that a spiritual needs assessment reveals important information for the treatment of, for example, schizophrenia [15], chronic kidney disease [16], diabetes [17], or heart failure [18]. Several studies emphasize the role of spiritual needs for medication adherence. A crosssectional study found that "spirituality, religiosity and personal beliefs were the only variables consistently associated with compliance to medication" and suggested that "adequately addressing these aspects on patient's care may lead to an improvement in adherence patterns" [18]. Another cross-sectional study showed that adherent patients presented higher intrinsic religiosity [11]. Patients who were less trusting that a "powerful other" will take care of them were more likely to use unnecessary drugs [10]. An interview study with 51 patients with hypertension concluded that hopeless patients "adhere insufficiently or drop out completely, only improving their adherence if something happens that gives them hope or strength, or if they find a medical team that provides enough emotional support" [9].

Research shows that the majority of patients have a strong interest in discussing spirituality in medical encounters [19], and yet this aspect of care tends to be ignored, except in some special fields such as palliative care where spiritual care is being more and more implemented. While $85 \%$ of primary care physicians think they should be aware of patients' spirituality, most would not initiate a talk about it except for dying patients [20]. For many physicians, spirituality is an area that makes them feel uncomfortable due to difficulties with spiritual language and worries about time, ethical boundaries, and reluctant reactions of the patient $[14,21,22]$. It has been suggested that acquiring basic skills in spiritual needs assessment should be part of medical training. 
Studies on spiritual care training for physicians draw positive conclusions about the effectiveness and acceptance of the training by patients and physicians [23, 24] and, in the United States, curriculums and objective structured clinical examinations (OSCEs) for spiritual care have already been established [25].

\section{Loneliness/social activity}

The perceived meaningfulness of life is strongly linked to the degree to which a person maintains social relationships [26]. In a survey among elderly people living in German nursing homes, "feeling connected with family" was the second most important spiritual need [27]. Lacking self-efficacy, especially 'spiritual health efficacy' relating to one's perceived ability to generate spiritually based faith and inner strength, proved to be a strong predictor for loneliness in old age $[28,29]$. 'Social loneliness', which refers to the objective degree of social isolation or number of social contacts, should be distinguished from emotional loneliness, which refers to the subjective feeling of being alone and which might also be the symptom of a psychological disease [30].

Unfortunately, many elderly people are not satisfied with their social relationships. About one-fifth of the patients aged $>65$ years in general practice feel often or occasionally lonely and 55\% of them would like to get support to increase their social activity, but only $15 \%$ of them ever talked to their GP about these issues [31, 32]. GPs rarely ask patients about loneliness and, if so, they do it indirectly due to a feeling of powerlessness and inability to provide adequate support [33]. However, several systematic reviews show that interventions to reduce loneliness in elderly people can be effective [34-36]. Loneliness in elderly people is a risk factor for increased disease burden involving cognitive impairment [37], pain, depression, and fatigue [38], and abuse of benzodiazepines [39]. Furthermore, loneliness is associated with delayed hospital stays and increased costs [40, 41]. However, not all elderly patients suffer from being lonely. 'Freedom' and 'independence' are positive dimensions attributed to loneliness [42, 43]. For general practice it is important not to avoid discussions about loneliness and to identify those patients who need support to increase their social activity.

\section{Self-care}

In this project we define self-care activities as "activities patients can perform on their own except taking medications to increase their well-being". The positive correlation between self-care and self-efficacy are well described, for example, in studies among individuals with type 2 diabetes [44]. Based on these studies, it may be summarized that self-efficacy is a mediation variable for self-care behavior associated with positive health outcomes [45]. This is also expressed by the concept of 'self-care self-efficacy' which is defined as "one's perceived ability to perform relevant self-care activities" and sometimes seen as a dimension of self-management [45]. According to Buck et al., patient's confidence in their ability to perform self-care is a strong predictor of physical and emotional quality of life [46].

A large number of health programs have been developed to improve patients' self-care ability. These are mostly disease-specific and often based on Orem's nursing theory of self-care $[45,47-51]$. This theory is based on the assumption that people have a natural ability for self-care, and nursing should focus on improving selfcare behavior. This aspect of self-efficacy is also considered by DMPs. As part of the DMPs, all patients should participate in training sessions which focus on diseasespecific knowledge and skills (e.g., correct use of inhaler devices for COPD, nutrition counseling for diabetes, and so on) [52].

In this study we aim to advise patients about self-care activities to enhance their self-care abilities for frequent symptoms in old age which are not alarming for a severe disease, e.g., knee pain in chronic osteoarthritis, chronic sleeping disorders, and so on. The self-care activities will focus on simple exercises (e.g., relaxation exercises, balance exercises) and on "home remedies".

Home remedies or folk remedies are not clearly defined and, as discussed by Parisius et al. [53], a scientific definition is lacking. They could be described as "a traditional therapy often utilizing natural products, nutritional supplements or physical measures. Its effectiveness may be supported by familial, local or culturally accepted stories or rituals" [54]. We decided to include home remedies as an element in our intervention because they are easy to adopt, and elderly people are generally used to home remedies for self-care [55]. The application of home remedies requires theoretical knowledge and practical skills and, thus, optimally corresponds to the self-care concept. It is hypothesized that the application of home remedies has a positive effect on self-efficacy.

\section{Objectives}

The aim of this exploratory study and the accompanying process evaluation is to assess the effectiveness and feasibility of interventions to strengthen patients' self-efficacy by exploring their spiritual needs and personal resources and by addressing loneliness and self-care if needed.

\section{Methods/design \\ Trial design}

Figure 2 illustrates the trial design. An open, exploratory, cluster-randomized controlled trial with general practices as the unit of randomization and a follow-up time of 6 months will be conducted. 


\section{Study setting}

The interventions will be implemented in GP practices in the wider area around Heidelberg and Tübingen, two university cities in the federal state of Baden-Württemberg in southern Germany.

\section{Eligibility criteria}

Inclusion criterion for GPs is participation in at least one DMP. Inclusion criteria for patients are: 1 ) age $\geq 70$ years; 2) $\geq 3$ chronic conditions defined by a previously published diagnosis list [56]; 3) $\geq 3$ medications on a longterm basis; 4) participation in at least one DMP; and 5) ability to give informed consent, to take part in the spiritual needs assessment, and to complete the questionnaires (if necessary with assistance by a person not involved in the study) based on the GP's personal assessment.

\section{Interventions}

Two types of interventions should be distinguished.

\section{Interventions delivered by GPs and medical assistants to patients}

Patients in the intervention group will receive one prolonged counseling session as part of the regular appointment for the DMP during which their GP explores their personal resources and spiritual needs, including the need for more social contact and self-care activities. Based on previous research, we assume that the spiritual needs assessment will take 10-15 min [23]. Trained medical assistants will inform all patients about social activities for seniors in the community and about nonpharmacological treatment alternatives and, if appropriate, set targets with the patients (another 10-15 min). Patients in the intervention group will receive a diary and be asked to document when and how they conduct social or self-care activities.

\section{Aids provided to the GP practices by the study}

Patients, GPs, and medical assistants in the intervention group will have access to a web portal developed by the study team with a collection of social activities for seniors in the region near the respective practice. Comprehensive search options (radius search, thematic filters, map screens) will facilitate the identification of suitable offers. Additionally, the social activities will be made available as a brochure in print form. Furthermore, information sheets developed by the study team will be provided to the practices and patients in the intervention group in paper-based and digital forms describing self-care activities (simple exercises and home remedies) to reduce frequent symptoms in old age which are not alarming for a severe disease. GPs and medical assistants will participate in a 4- to 5-h workshop conceptualized and led by the authors of this protocol. For GPs, the workshop will comprise an introduction to the definition of spirituality, the concept of spiritual care, and a previously evaluated conversation technique (SPIR [23]), as well as self-reflection of their own spirituality and practical exercises on conducting a spiritual needs assessment based on videos of real patients and role plays with trained actors. The training of medical assistants will focus on patient counseling regarding self-care and social activity and on the handling of the web portal and the info sheets. Possible barriers and facilitators for implementing the HoPES3 program into practice will be discussed.

\section{Control}

In the control practices, DMPs will be conducted as usual. Although DMPs are defined by a standardized treatment form to be completed during each patient contact, the way DMPs are organized in GP practices varies substantially. To get a better idea of usual care in our study, we will ask all participating GPs and medical assistants to describe how they usually organize DMPs in their practice.

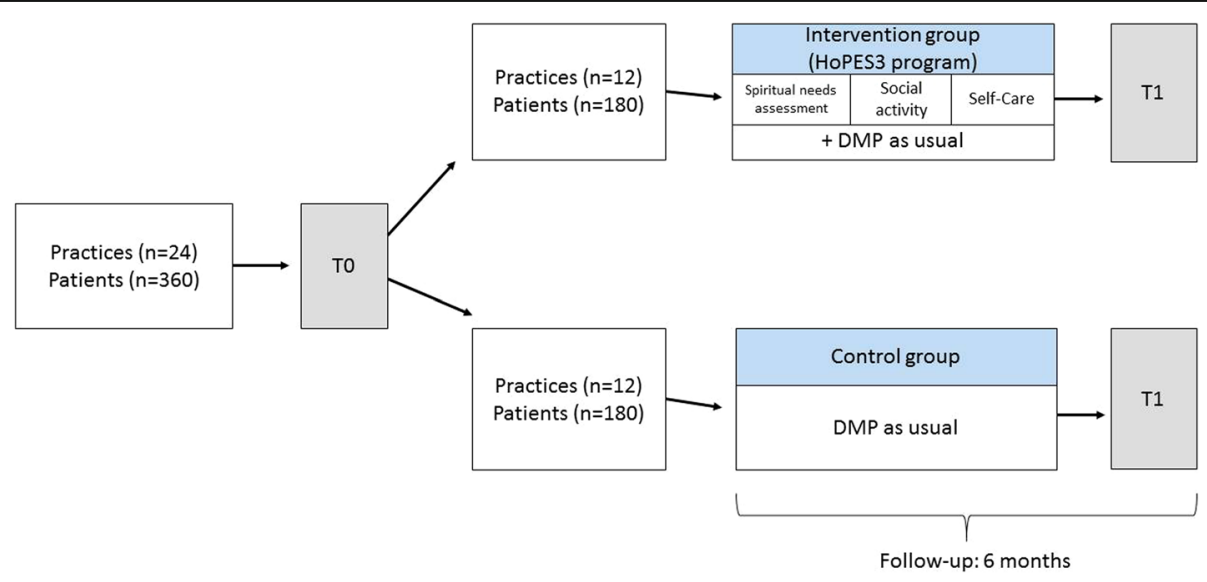

Fig. 2 Trial design of the HoPES3 study. DMP disease management program 


\section{Outcomes}

The primary and secondary outcomes of this study will be based on primary data collected in written, paper-based surveys. The rationale for choosing the various outcomes is shown schematically in Fig. 1. The primary outcome is patients' self-efficacy measured by the Self-Efficacy for Managing Chronic Disease 6-Item Scale (SES6G). The German version of the SES6G showed good construct validity and high internal consistency [57]. In addition to health-related self-efficacy, general self-efficacy will be measured by the General Self-Efficacy Scale (GSES) [58].

Secondary outcomes were chosen to assess the effects of each of the single intervention components and of the entire HoPES3 Program. To assess the patients' social activity, the short, six-item version of the Lubben Social Network Scale (LSNS-6) [59] and the 11-item De-Jong-Gierveld Loneliness Scale (DJGS-11) [30] will be used. The LSNS-6 determines the number of available contact persons (i.e., the degree of social isolation) and the DJGS-11 measures the perceived social and emotional loneliness. Patients' self-care abilities will be estimated by the Patient Activation Measure (PAM) [60]. Awareness of own spirituality or the patients' spirituality, respectively, will be assessed by selfdeveloped items.

We hypothesize that the HoPES3 program will improve patient quality of life as assessed by the Short Form Health Survey (SF-12) [61], and satisfaction with GP care and patient-centered communication will be assessed by selected items of the European Project on Patient Evaluation of General Practice (EUROPEP) questionnaire [62]. To explore whether HoPES3 has any influence on the use of medications, the Medication Adherence Report Scale (MARS) [11] and the general part of Beliefs About Medicines Questionnaire (BMQ) [63] will be used. At the level of GPs and medical assistants, work satisfaction (using the Warr-Cook-Wall-Scale) [64], competence in providing spiritual care (using the Spiritual Care Competence Questionnaire) [65], or in advising self-care activities and awareness of own spirituality (using self-developed items) will be assessed.

\section{Other data}

Besides outcome measures, descriptive data and process data will be collected. We will use the Spiritual Needs Questionnaire [66] and self-developed items to describe the type and intensity of the spiritual needs of the sample and whether their spirituality is a source of strength or rather a risk factor to them. Furthermore, we will collect printouts of the diagnosis lists and medication lists saved in the patient records of the participating practices. We will ask GPs, medical assistants, and patients in the intervention group to assess the usefulness of and strain caused by the interventions within 2 weeks after the spiritual needs assessment via a short self-developed questionnaire. A diary will be given to patients in the intervention group asking them to document how often and what type of social and self-care activities they apply. The log file of the web portal will be analyzed to determine which functions were used and how often.

After the intervention period terminates, interviews with GPs, medical assistants, and patients in the intervention group will be conducted, audiotaped, and transcribed for the purpose of a comprehensive process evaluation. Research questions of the process evaluation are:

- Intervention fidelity

- Were the interventions delivered as specified in the protocol?

- How is the acceptance of the interventions by GPs, medical assistants, and patients?

- How feasible are the interventions for use in daily practice?

- What are potential barriers and solutions for delivering the interventions as intended?

- Intervention mechanisms

- What is the perceived benefit of the interventions for patients and healthcare professionals? What are potential harms?

- Which subgroups of patients profit from the intervention, and which do not?

- How did the interventions influence the patientphysician relationship?

- How did the interventions influence medical treatment, especially medication adherence and use of unnecessary drugs?

- Intervention costs

- How big is the effort for performing the interventions?

\section{Recruitment and participant timeline}

The time schedule of the study is depicted in Fig. 3. GPs offering DMPs will be recruited in the wider area of Heidelberg and Tübingen. For this purpose, the study information will be sent to the practices of already established research networks of the University Hospitals Heidelberg and Tübingen. Participating GPs will identify all patients meeting the eligibility criteria using the filter options of their practice software. GPs will be asked to estimate which patients would profit most from the HoPES3 program and to include 15 of these patients into the study. The reasons for selecting or excluding patients as well as for refusing to participate will be documented and explored in the process evaluation. All participants will give written informed consent. In case of patients, the informed consent forms will remain in the practices. 


\begin{tabular}{|c|c|c|c|c|c|c|c|c|}
\hline \multirow{4}{*}{$\begin{array}{c}\text { Month } \\
\text { TIMEPOINT }\end{array}$} & \multicolumn{8}{|c|}{ Study period } \\
\hline & \multirow{3}{*}{$\begin{array}{c}\text { Enrolment } \\
\text { Month 1-8 } \\
-t_{1}\end{array}$} & \multirow{3}{*}{$\begin{array}{c}\text { Allocation } \\
\text { Month } 8 \\
0\end{array}$} & \multicolumn{4}{|c|}{ Post-allocation } & \multicolumn{2}{|c|}{ Close-out } \\
\hline & & & \multirow[t]{2}{*}{ Month 9} & \multicolumn{3}{|c|}{ Month 10-15 } & \multirow{2}{*}{$\begin{array}{c}\text { Month } \\
15-17 \\
t_{3} \\
\end{array}$} & \multirow{2}{*}{$\begin{array}{c}\text { Month } \\
18-20 \\
t_{4}\end{array}$} \\
\hline & & & & $t_{1}$ & $t_{2}$ & & & \\
\hline \multicolumn{9}{|l|}{ ENROLMENT: } \\
\hline \multirow{2}{*}{$\begin{array}{r}\text { Intervention development } \\
\text { Recruitment of GPs and MAs + } \\
\text { informed consent }\end{array}$} & $x$ & & & & & & & \\
\hline & $x$ & & & & & & & \\
\hline \multirow[t]{2}{*}{$\begin{array}{r}\text { Recruitment of patients }+ \\
\text { informed consent }\end{array}$} & $\mathrm{x}$ & & & & & & & \\
\hline & $\mathrm{x}$ & & & & & & & \\
\hline ALLOCATION & & $\mathrm{x}$ & & & & & & \\
\hline \multicolumn{9}{|l|}{ INTERVENTIONS: } \\
\hline Training of GPs and MAs & & & $\mathrm{x}$ & & & & & \\
\hline \multicolumn{8}{|l|}{$\begin{array}{r}\text { Spiritual needs assessment + } \\
\text { goal setting }\end{array}$} & \\
\hline \multicolumn{9}{|l|}{ Follow-up goals } \\
\hline \multicolumn{9}{|l|}{ ASSESSMENTS: } \\
\hline \multicolumn{9}{|l|}{ Outcome assessment GPs: } \\
\hline$S C C Q$ & $x$ & & & & & & $\mathrm{x}$ & \\
\hline wCWs & $x$ & & & & & & $x$ & \\
\hline \multirow{3}{*}{$\begin{array}{c}\text { Awareness of own spirituality } \\
\text { Dealing with lonely patients } \\
\text { Use of home remedies }\end{array}$} & $x$ & & & & & & $x$ & \\
\hline & $\mathrm{X}$ & & & & & & $\mathrm{x}$ & \\
\hline & $\mathrm{x}$ & & & & & & $\mathrm{x}$ & \\
\hline \multicolumn{9}{|l|}{ Outcome assessment MAs: } \\
\hline \multirow{3}{*}{$\begin{array}{l}\text { WCWS } \\
\text { Awareness of own spirituality* } \\
\text { Dealing with lonely patients }\end{array}$} & $\mathrm{x}$ & & & & & & $\mathrm{x}$ & \\
\hline & $\mathrm{X}$ & & & & & & $\mathrm{X}$ & \\
\hline & $\mathrm{x}$ & & & & & & $x$ & \\
\hline Use of home remedies ${ }^{*}$ & $x$ & & & & & & $x$ & \\
\hline \multicolumn{9}{|l|}{$\begin{array}{r}\text { Outcome assessment } \\
\text { patients: }\end{array}$} \\
\hline SES6G & $\mathrm{X}$ & & & & & & $\mathrm{X}$ & \\
\hline GSES & $\mathrm{x}$ & & & & & & $\mathrm{X}$ & \\
\hline LSNS-6 & $\mathrm{x}$ & & & & & & $\mathrm{X}$ & \\
\hline DJGS-11 & $x$ & & & & & & $x$ & \\
\hline PAM & $\mathrm{x}$ & & & & & & $\mathrm{x}$ & \\
\hline SF-12 & $x$ & & & & & & $x$ & \\
\hline$B M Q$ & $\mathrm{x}$ & & & & & & $\mathrm{x}$ & \\
\hline \multirow{3}{*}{$\begin{array}{r}\text { MARS } \\
\text { Awareness of own spirituality* } \\
\text { EUROPEP }\end{array}$} & $\mathrm{X}$ & & & & & & $x$ & \\
\hline & $\mathrm{X}$ & & & & & & $\mathrm{X}$ & \\
\hline & $\mathrm{X}$ & & & & & & $\mathrm{x}$ & \\
\hline \multirow{4}{*}{$\begin{array}{r}\text { Process evaluation } \\
\text { Patient survey } \\
\text { Survey of MAs } \\
\text { Survey of GPs } \\
\text { terviews with GPs, MAs and } \\
\text { patients of intervention group }\end{array}$} & & & & $x$ & & & & \\
\hline & & & & $\mathrm{X}$ & $\mathrm{X}$ & & & \\
\hline & & & & $\mathrm{x}$ & $\mathrm{x}$ & & & \\
\hline & & & & & & & & $\mathrm{x}$ \\
\hline
\end{tabular}

Fig. 3 Time schedule of the HoPES3 study (following the SPIRIT recommendations). BMQ Beliefs About Medicines Questionnaire, DJGS-11 11-Item De-Jong-Gierveld Loneliness Scale, EUROPEP European Project on Patient Evaluation of General Practice, GP general practitioner, GSES General Self-Efficacy Scale, LSNS-6 6-Item Lubben Social Network Scale, MA medical Assistant, MARS Medication Adherence Report Scale, PAM Patient Activation Measure, SES6G Self-Efficacy for Managing Chronic Disease 6-Item Scale, SCCQ Spiritual Care Competence Questionnaire, SF-12 ShortForm Health Survey, WCWS Warr-Cook-Wall Scale

\section{Management, collection, and monitoring of data}

All data will be managed at the study center located at the University Hospital Heidelberg, Department of General Practice and Health Services Research. The study center will receive patient data only in pseudonymized form and will not have direct contact with patients (except for interviews with patients who gave their permission to be contacted by the study team). Only authorized staff members are able to enter or edit data. For specific research questions, parts of the data will be made available to partners of the project (University Hospitals Munich and Tübingen) in pseudonymous form after the trial biometrician has finalized the statistical report. The partners guarantee that only the analyzing researchers will have access to the data. It is also planned to make trial data on which scientific publications are based and all the primary data publicly available for re- and meta-analyses after the trial has been completed.

A study nurse will monitor the data entry during the entire study period. Furthermore, the study team will 
visit all participating practices before randomization to explain organizational issues related to the study. A Data Safety and Monitoring Board (DSMB) consisting of a GP, a statistician, and an expert in spiritual care will be regularly informed about the course of the trial and all safety issues, and asked for advice whether to continue, modify, or stop the trial.

\section{Sample size}

The sample size was calculated using the primary endpoint, the SES6G at T1. With $n=22$ practices (11 per group) and $n=264$ (132 per group) patients available for analysis, it will be possible to detect a clinically relevant mean difference of $\delta=1$ point at T1 between the two treatment groups for the mean SES6G score at a twosided $\alpha$ of 0.05 with a power of $1-\beta=0.8$, assuming a standard deviation of $\sigma=2.2$ based on the results of Lorig et al. [67], a relatively high intraclass correlation coefficient [68] of 0.05 , and a cluster size of $m=12$ (the calculation was done using the function n4means from the $\mathrm{R}$ package CRTSize). It is expected that, adjusting for the SES6G score at T0, gender, age, number of comorbidities, and number of medications in the linear mixed model used for statistical analysis will lead to less unexplained variance and thus to an increase in power. Taking drop-out rates of $7.5 \%$ at the practice level and $20 \%$ at the patient level, respectively, into account, $n=$ 24 practices with $n=360$ patients will be enrolled into the trial. The potential problem of missing values for drop outs will be partly resolved in the primary analysis by application of the pre-defined imputation strategy.

\section{Statistical methods}

The primary objective of this study is to determine the effectiveness of a complex intervention compared with usual care. The primary efficacy endpoint is the SES6G score at T1. The study objective is statistically formulated as a test of the null hypothesis $\mathrm{H}_{0}: \mu_{\mathrm{I}}=\mu_{\mathrm{C}}$ (the mean SES6G score at $\mathrm{T} 1$ in the intervention and the control group are equal) against its alternative $\mathrm{H}_{1}: \mu_{\mathrm{I}} \neq \mu_{\mathrm{C}}$ at a significance level of $\alpha=0.05$ (two-sided).

Because of the cluster randomization, the primary efficacy analysis will use a multilevel regression approach with patients at level one and practices at level two. The primary efficacy analysis will be performed by fitting a linear mixed model including the SES6G score at T1 as the dependent variable, treatment group and gender as fixed factors, and SES6G score at T0, age, number of comorbidities, and number of medications as fixed covariates, and practice as a random factor to account for the two-level data structure (patients nested within practices). The results will be presented as the mean between-group difference in SES6G at T1 with the corresponding 95\% confidence interval. The associated Cohen's effect size $d$ will be calculated. In addition, the practice-related intracluster correlation coefficient (ICC) will be estimated. The primary analysis will be performed adhering to the intention-to-treat principle. An additional sensitivity analysis will be conducted on the perprotocol analysis set. Missing data for the primary outcome variable will be replaced using multiple imputation [69] which takes the covariates of treatment group, gender, age, number of comorbidities, number of medications, and practice into account by application of the fully conditional specification method [70]. Sensitivity analyses will be performed by applying alternative methods dealing with missing data such as, for example, complete case analysis and best/worst case imputation.

The statistical analyses of the secondary endpoints will use the same multilevel approach as the primary analysis. All statistical tests will be two-sided at the significance level of $\alpha=0.05$. Because no adjustments for multiple endpoints are planned, findings will be interpreted with caution in view of the number of statistical tests undertaken. Only the result of the primary efficacy analysis will be interpreted in a confirmatory manner. Confirmatory subgroup analyses are not planned. No interim analysis with regard to efficacy will be done. All analyses will be performed using SAS version 9.4 or higher.

\section{Assignment of interventions}

As Fig. 2 shows, 360 patients and 24 practices will be recruited (half in the area of Heidelberg, half in the area of Tübingen) and randomized to either intervention or control group using block randomization stratified by region (Heidelberg/Tübingen) to ensure an equal number of intervention and control practices per region. The randomization list will be created by the trial statistician. Since the intervention involves training of GPs and informed consent of patients, blinding of participants is not possible. However, acknowledged measures to reduce bias such as computerized randomization and blinding of outcome assessment will be undertaken.

\section{Harms}

The pharmacological treatment of the patients is not altered by the study, and therefore we do not expect severe risks to health. However, it is possible that the spiritual needs assessment causes psychological strain for the participating patients and/or GPs. Although the strain caused by the conversation technique (SPIR) that will be applied in this study was considered low in previous studies, we will ask patients and GPs to evaluate the strain within 2 weeks after each assessment by means of a questionnaire. The responses of each patient and those aggregated at a practice level will be discussed with the DSMB. 


\section{Discussion}

To our knowledge, HoPES3 is the first study examining interventions to address spiritual needs, social activity, and self-care in elderly patients in German general practice.

Since little is known about the effectiveness of such interventions, HoPES3 is designed as an explorative trial. This means we have chosen a range of secondary outcomes beside the primary outcome to assess the possible effects of the program. If the program proves to be effective and feasible, large-scale implementation should be sought and evaluated by confirmatory research. This could be done, for instance, by integrating the training concept into the university curricula for medical students or into continuous education of GPs or by collaborating with health insurance companies or local state authorities.

In German language the term spirituality is frequently associated with esotericism or religion. We are aware that some GPs and maybe also patients might have a negative attitude towards this topic. Therefore, we will explain in the invitation letter and study information how spirituality is defined in our project and that a spiritual needs assessment is a way of resource-oriented communication which has the potential to reveal important information for the treatment of the patient. However, we cannot exclude that there will be a selection bias in favor of those GPs and patients who are already interested in the topic and assess this aspect during the baseline assessment.

In this study, GPs will select those patients who, in their opinion, will profit most from the intervention. While this approach may be criticized in clinical research and efficacy trials because it limits the generalizability of the findings, it is justifiable in health services research which focusses on the effectiveness of interventions under real-life conditions. It is common in general practice that GPs decide which treatment or which conversation technique is appropriate for which patient. Therefore, we believe that the selection of patients by GPs is a realistic approach which would also be applied in case the HoPES3 program is implemented into routine care. The reasons for selecting or excluding patients will be examined and will reveal important information for the adaption and dissemination of the program.

\footnotetext{
Abbreviations

BMQ: Beliefs About Medicines Questionnaire; DJGS-11: 11-Item De-JongGierveld Loneliness Scale; DMP: Disease management program; DRKS: German Registry for Clinical Trials; EUROPEP: European Project on Patient Evaluation of General Practice; GP: General practitioner; GSES: General Self-Efficacy Scale; LSNS-6: 6-Item Lubben Social Network Scale; MARS: Medication Adherence Report Scale; PAM: Patient Activation Measure; SES6G: Self-Efficacy for Managing Chronic Disease 6-Item Scale; SF-12: ShortForm Health Survey
}

\section{Acknowledgements}

The authors thank the self-help organization Gesundheitstreffpunkt Mannheim e.V. for supporting their project.

\section{Trial status}

The funding period of the trial started on 1 October 2018 and will end on 30 September 2020. Recruitment of GPs is planned to start in February 2019 and recruitment of patients in March 2019. The intervention period will start with the workshop for practice teams in June 2019 and end with the T1 data collection (last patient out) in February 2020. Protocol version V1 dated 18 January 2019

\section{Authors' contributions}

CS, SJ, and EF conceptualized the design of the trial and interventions.All authors except JK are involved into the final elaboration of the intervention components. JK conducted the sample size calculation and wrote the section on statistical methods. CS wrote the first draft of this article. EF, GS, NB, RS, FS, JV, SJ, JK and JZ critically revised it. All authors read and approved the final version of the manuscript.

\section{Funding}

The trial is funded by the German Federal Ministry of Education and Research (funding code 01GL1803).

\section{Availability of data and materials}

Not applicable.

\section{Ethics approval and consent to participate}

This study was approved by the ethics committee of the Medical Faculty of the University Hospital Heidelberg (S-730/2018). The study will be carried out in compliance with the Declaration of Helsinki (2008 version, Seoul, Korea) and local legal regulations. All participants will be asked to provide their written informed consent prior to participation in the study.

\section{Consent for publication}

Not applicable.

\section{Competing interests}

The authors declare that they have no competing interests.

\section{Author details}

'Department of General Practice and Health Services Research, University Hospital Heidelberg, Im Neuenheimer Feld 130.3, 69120 Heidelberg, Germany. ${ }^{2}$ Department of Psychosomatic Medicine and Psychotherapy, Research Center Spiritual Care, Technical University of Munich, Langerstr. 3, 81675 München, Germany. ${ }^{3}$ Department for Medical Biometry, Institute for Medical Biometry and Informatics, University Hospital Heidelberg, Im Neuenheimer Feld 130.3, 69120 Heidelberg, Germany. ${ }^{4}$ Institute of General Practice and Interprofessional Care, University Hospital Tübingen, Osianderstr. 5, 72076 Tübingen, Germany.

Received: 22 February 2019 Accepted: 13 May 2019

Published online: 18 June 2019

\section{References}

1. Bundeszentrale für Politische Bildung (German Federal Agency for Civic Education). [Europe's demographic future]. http://www.bpb.de/politik/ innenpolitik/demografischer-wandel/196906/europas-demografische-zukunft (Accessed 18 Jan 2019).

2. Midao L, Giardini A, Menditto E, Kardas P, Costa E. Polypharmacy prevalence among older adults based on the survey of health, ageing and retirement in Europe. Arch Gerontol Geriatr. 2018;78:213-20.

3. Hakkarainen KM, Hedna K, Petzold M, Hagg S. Percentage of patients with preventable adverse drug reactions and preventability of adverse drug reactions - a meta-analysis. PLoS One. 2012;7(3):e33236.

4. Hanlon P, Nicholl BI, Jani BD, McQueenie R, Lee D, Gallacher Kl, et al. Examining patterns of multimorbidity, polypharmacy and risk of adverse drug reactions in chronic obstructive pulmonary disease: a cross-sectional UK Biobank study. BMJ Open. 2018;8(1):e018404. 
5. Meraya AM, Dwibedi N, Sambamoorthi U. Polypharmacy and health-related quality of life among US adults with arthritis, medical expenditure panel survey, 2010-2012. Prev Chronic Dis. 2016;13:E132.

6. Labella AM, Merel SE, Phelan EA. Ten ways to improve the care of elderly patients in the hospital. J Hosp Med. 2011;6(6):351-7.

7. Rankin A, Cadogan CA, Patterson SM, Kerse N, Cardwell CR, Bradley MC, et al. Interventions to improve the appropriate use of polypharmacy for older people. Cochrane Database Syst Rev. 2018;9:CD008165.

8. Halisch F, Geppert U. Well-being in old age: the influence of self-efficacy, control beliefs, coping strategies, and personal goals. Results from the Munich Gold Study. http://psydok.psycharchives.de/jspui/handle/20.500. 11780/1955 (Accessed 18 Jan 2019).

9. Herrera PA, Moncada L, Defey D. Understanding non-adherence from the inside: hypertensive patients' motivations for adhering and not adhering. Qual Health Res. 2017;27(7):1023-34.

10. Rossi MI, Young A, Maher R, Rodriguez KL, Appelt CJ, Perera S, et al. Polypharmacy and health beliefs in older outpatients. Am J Geriatr Pharmacother. 2007:5(4):317-23.

11. Silva AN, Moratelli L, Tavares PL, Marsicano EO, Pinhati RR, Colugnati FA, et al. Self-efficacy beliefs, locus of control, religiosity and non-adherence to immunosuppressive medications in kidney transplant patients. Nephrology. 2016;21(11):938-43.

12. Weathers E, McCarthy G, Coffey A. Concept analysis of spirituality: an evolutionary approach. Nurs Forum. 2016;51(2):79-96.

13. Fegg MJ, Kramer M, Bausewein C, Borasio GD. Meaning in life in the Federal Republic of Germany: results of a representative survey with the Schedule for Meaning in Life Evaluation (SMiLE). Health Qual Life Outcomes. 2007:5:59.

14. Koenig HG. Religion, spirituality, and health: a review and update. Adv Mind Body Med. 2015;29(3):19-26.

15. Huguelet $P$, Mohr S, Betrisey C, Borras L, Gillieron C, Marie AM, et al. A randomized trial of spiritual assessment of outpatients with schizophrenia: patients' and clinicians' experience. Psychiatr Serv. 2011;62(1):79-86.

16. Fradelos EC, Tzavella F, Koukia E, Papathanasiou IV, Alikari V, Stathoulis $J$, et al. Integrating chronic kidney disease patient's spirituality in their care: health benefits and research perspectives. Mater Sociomed. 2015; 27(5):354-8

17. Watkins YJ, Quinn LT, Ruggiero L, Quinn MT, Choi YK. Spiritual and religious beliefs and practices and social support's relationship to diabetes self-care activities in African Americans. Diabetes Educ. 2013;39(2):231-9.

18. Alvarez JS, Goldraich LA, Nunes AH, Zandavalli MC, Zandavalli RB, Belli KC, et al. Association between spirituality and adherence to management in outpatients with heart failure. Arq Bras Cardiol. 2016;106(6):491-501.

19. Best M, Butow P, Olver I. Do patients want doctors to talk about spirituality? A systematic literature review. Patient Educ Couns. 2015; 98(11):1320-8

20. Monroe MH, Bynum D, Susi B, Phifer N, Schultz L, Franco M, et al. Primary care physician preferences regarding spiritual behavior in medical practice. Archiv Intern Med. 2003;163(22):2751-6.

21. Best M, Butow P, Olver I. Why do we find it so hard to discuss spirituality? A qualitative exploration of attitudinal barriers. J Clin Med. 2016:5(9):E77.

22. Ellis MR, Campbell JD, Detwiler-Breidenbach A, Hubbard DK. What do family physicians think about spirituality in clinical practice? J Fam Pract. 2002; 51(3):249-54.

23. Frick E, Riedner C, Fegg MJ, Hauf S, Borasio GD. A clinical interview assessing cancer patients' spiritual needs and preferences. Eur J Cancer Care. 2006;15(3):238-43.

24. Pettus MC. Implementing a medicine-spirituality curriculum in a communitybased internal medicine residency program. Acad Med. 2002;77(7):745.

25. Ledford CJ, Seehusen DA, Canzona MR, Cafferty LA. Using a teaching OSCE to prompt learners to engage with patients who talk about religion and/or spirituality. Acad Med. 2014;89(1):60-5.

26. Früh J. Auswirkungen palliativmedizinischer Interventionen auf den Lebenssinn, gemessen mit dem SMiLE. Dissertation. Würzburg; 2015. https:// opus.bibliothek.uni-wuerzburg.de/files/13457/Dissertation_Frueh_Jonas.pdf (Accessed 21 Jan 2019)

27. Erichsen NB, Bussing A. Spiritual needs of elderly living in residential/nursing homes. Evid Based Complement Alternat Med. 2013;2013:913247.

28. Cohen-Mansfield J, Hazan H, Lerman Y, Shalom V. Correlates and predictors of loneliness in older-adults: a review of quantitative results informed by qualitative insights. Int Psychogeriatr. 2016;28(4):557-76.
29. Fry PS, Debats DL. Self-efficacy beliefs as predictors of loneliness and psychological distress in older adults. Int J Aging Hum Dev. 2002;55(3): 233-69.

30. De Jong Gierveld J, Van Tilburg T. The De Jong Gierveld short scales for emotional and social loneliness: tested on data from 7 countries in the UN generations and gender surveys. Eur J Ageing. 2010;7(2):121-30.

31. Due TD, Sandholdt H, Waldorff FB. Social relations and loneliness among older patients consulting their general practitioner. Dan Med J. 2017;64(3).

32. Hand C, McColl MA, Birtwhistle R, Kotecha JA, Batchelor D, Barber KH. Social isolation in older adults who are frequent users of primary care services. Can Fam Physician. 2014;60(6):e322 e4-9.

33. van Ravesteijn H, Lucassen P, van der Akker M. GPs' experiences with loneliness. Aus Fam Physician. 2008;37(11):973-6.

34. Dickens AP, Richards SH, Greaves CJ, Campbell JL. Interventions targeting social isolation in older people: a systematic review. BMC Public Health. 2011;11:647

35. Franck L, Molyneux N, Parkinson L. Systematic review of interventions addressing social isolation and depression in aged care clients. Qual Life Res. 2016;25(6):1395-407.

36. Hagan R, Manktelow R, Taylor BJ, Mallett J. Reducing loneliness amongst older people: a systematic search and narrative review. Aging Ment Health. 2014;18(6):683-93.

37. Boss L, Kang DH, Branson S. Loneliness and cognitive function in the older adult: a systematic review. Int Psychogeriatr. 2015;27(4):541-53.

38. Jaremka LM, Andridge RR, Fagundes CP, Alfano CM, Povoski SP, et al. Pain, depression, and fatigue: loneliness as a longitudinal risk factor. Health Psychol. 2014;33(9):948-57.

39. Canham SL. What's loneliness got to do with it? Older women who use benzodiazepines. Australas J Ageing. 2015;34(1):E7-E12.

40. Hawker M, Romero-Ortuno R. Social determinants of discharge outcomes in older people admitted to a geriatric medicine ward. J Frailty Aging. 2016; 5(2):118-20

41. Landeiro F, Leal J, Gray AM. The impact of social isolation on delayed hospital discharges of older hip fracture patients and associated costs. Osteoporos Int. 2016;27(2):737-45.

42. Kirkevold M, Moyle W, Wilkinson C, Meyer J, Hauge S. Facing the challenge of adapting to a life 'alone' in old age: the influence of losses. J Adv Nurs. 2013;69(2):394-403.

43. Taube $E$, Jakobsson U, Midlov P, Kristensson J. Being in a bubble: the experience of loneliness among frail older people. J Adv Nurs. 2016; 72(3):631-40.

44. Tharek Z, Ramli AS, Whitford DL, Ismail Z, Mohd Zulkifli M, Ahmad Sharoni SK, et al. Relationship between self-efficacy, self-care behaviour and glycaemic control among patients with type 2 diabetes mellitus in the Malaysian primary care setting. BMC Fam Pract. 2018;19(1):39.

45. Eller LS, Lev EL, Yuan C, Watkins AV. Describing self-care self-efficacy: definition, measurement, outcomes, and implications. Int J Nurs Knowl. 2018;29(1):38-48.

46. Buck HG, Lee CS, Moser DK, Albert NM, Lennie T, Bentley B, et al. Relationship between self-care and health-related quality of life in older adults with moderate to advanced heart failure. J Cardiovasc Nurs. 2012; 27(1):8-15.

47. Kusnanto K, Sari N, Harmayetty H, Efendi F, Gunawan J. Self-care model application to improve self-care agency, self-care activities, and quality of life in patients with systemic lupus erythematosus. J Taibah Univ Med Sci. 2018;13(5):472-8.

48. Saeedifar ES, Memarian R, Fatahi S, Ghelichkhani F. Use of the Orem selfcare model on pain relief in women with rheumatoid arthritis: a randomized trial. Electron Physician. 2018;10(6):6884-91.

49. Mahmoudzadeh-Zarandi F, Hamedanizadeh F, Ebadi A, Raiesifar A. The effectiveness of Orem's self-care program on headache-related disability in migraine patients. Iran J Neurol. 2016;15(4):240-7.

50. Mohammadpour A, Rahmati Sharghi N, Khosravan S, Alami A, Akhond M. The effect of a supportive educational intervention developed based on the Orem's self-care theory on the self-care ability of patients with myocardial infarction: a randomised controlled trial. J Clin Nurs. 2015;24(11-12):1686-92.

51. Altay N, Cavusoglu H. Using Orem's self-care model for asthmatic adolescents. J Spec Pediatr Nurs. 2013;18(3):233-42.

52. Kassenärztliche Vereinigung Baden-Württemberg (KVBW). Disease Management Programs. https://www.kvbawue.de/praxis/neueversorgungsmodelle/dmp/ (Accessed 18 Jan 2019). 
53. Parisius LM, Stock-Schroer B, Berger S, Hermann K, Joos S. Use of home remedies: a cross-sectional survey of patients in Germany. BMC Fam Pract. 2014;15:116.

54. Medical Dictionary. 2009. https://medical-dictionary.thefreedictionary.com/ Folk+remedies (Accessed 18 Jan 2019).

55. Sackett K, Carter M, Stanton M. Elders' use of folk medicine and complementary and alternative therapies: an integrative review with implications for case managers. Prof Case Manag. 2014;19(3):113-23 quiz 24-5.

56. Altiner A, Schafer I, Mellert C, Loffler C, Mortsiefer A, Ernst A, et al. Activating GENeral practitioners dialogue with patients on their Agenda (MultiCare AGENDA) study protocol for a cluster randomized controlled trial. BMC Fam Pract. 2012;13:118.

57. Freund T, Gensichen J, Goetz K, Szecsenyi J, Mahler C. Evaluating selfefficacy for managing chronic disease: psychometric properties of the sixitem Self-Efficacy Scale in Germany. J Eval Clin Pract. 2013;19(1):39-43.

58. Schwarzer R, Jerusalem M. Generalized Self-Efficacy scale. In: Weinman J, Wright S, Johnston M, editors. Measures in health psychology: a user's portfolio causal and control beliefs. Windsor: NFER-NELSON; 1995. p. 35-7.

59. Lubben J, Blozik E, Gillmann G, lliffe S, von Renteln Kruse W, Beck JC, et al. Performance of an abbreviated version of the Lubben Social Network Scale among three European community-dwelling older adult populations. Gerontologist. 2006;46(4):503-13.

60. Brenk-Franz K, Hibbard JH, Herrmann WJ, Freund T, Szecsenyi J, Djalali S, et al. Validation of the German version of the patient activation measure 13 (PAM13-D) in an international multicentre study of primary care patients. PLoS One. 2013;8(9):e74786.

61. Ware J, Kosinski M, Keller SD. A 12-Item Short-Form Health Survey: construction of scales and preliminary tests of reliability and validity. Med Care. 1996;34(3):220-33.

62. Wensing M, Mainz J. R G. A standardised instrument for patient evaluations of general practice care in Europe. Eur J Gen Pract. 2000;6(3):82-7.

63. Horne R, Weinman J, Hankins M. The beliefs about medicines questionnaire: the development and evaluation of a new method for assessing the cognitive representation of medication. Psychol Health. 1999;14:1-24.

64. Heritag B, Pollock C, Roberts LD. Confirmatory factor analysis of Warr, Cook, and Wall's (1979) Job Satisfaction Scale. Aust Psychol. 2015;50(2):122-12.

65. Frick E, Theiss M, Rodrigues Recchia D, Büssing A. Validierung einder deutschsprachigen Skala zur Messung der Spiritual Care - Kompetenz. Spiritual Care. 2019; in press.

66. Bussing A, Balzat HJ, Heusser P. Spiritual needs of patients with chronic pain diseases and cancer-validation of the spiritual needs questionnaire. Eur J Med Res. 2010;15(6):266-73.

67. Lorig KR, Sobel DS, Ritter PL, Laurent D, Hobbs M. Effect of a selfmanagement program on patients with chronic disease. Eff Clin Pract. 2001; 4(6):256-62

68. Bonacchi A, Toccafondi A, Mambrini A, Cantore M, Muraca MG, Focardi F, et al. Complementary needs behind complementary therapies in cancer patients. Psychooncology. 2015;24(9):1124-30.

69. van Buuren S. Flexible imputation of missing data: Chapman \& Hall/CRC Interdisciplinary Statistics 2012: Chapman and Hall/CRC; 2012.

70. van Buuren S. Multiple imputation of discrete and continuous data by fully conditional specification. Stat Methods Med Res. 2007;16(3):219-42.

\section{Publisher's Note}

Springer Nature remains neutral with regard to jurisdictional claims in published maps and institutional affiliations.

Ready to submit your research? Choose BMC and benefit from:

- fast, convenient online submission

- thorough peer review by experienced researchers in your field

- rapid publication on acceptance

- support for research data, including large and complex data types

- gold Open Access which fosters wider collaboration and increased citations

- maximum visibility for your research: over $100 \mathrm{M}$ website views per year

At BMC, research is always in progress.

Learn more biomedcentral.com/submissions 